\title{
Evaluación de herramientas tecnológicas de uso libre, aplicadas a procesos de auditoría
}

\author{
Evaluation of free use technology software, applied to audit process \\ Laura Constanza Gallego Cossío, Ludivia Hernández Aros, Nelly Clavijo Bustos \\ Facultad de Contaduría Pública, Universidad Cooperativa de Colombia, Ibagué, Colombia \\ Correo-e ${ }^{1}$ : laura.gallegolcampusucc.edu.co \\ Correo- $e^{2}$ : lauracoss23ehotmail.com \\ Correo-e $e^{3}$ lauracoss2360@gmail.com
}

\begin{abstract}
Resumen- Este artículo sistematiza los resultados de la investigación titulada "Impacto de procesos de auditoria en la fase de planeación bajo conceptos de técnicas especializadas de análisis de datos en las empresas de la ciudad de Ibagué" cuya pretensión es establecer comparaciones que permitan formar juicios sobre la utilización de software libre para una aplicación específica, lo que mitológicamente se desarrolla mediante un análisis comparativo, entre las herramientas o software con licencia pública más utilizadas para el desarrollo de una auditoría financiera.

Estructuralmente consta de tres apartados, el primero de ellos sistematiza la literatura de las técnicas y herramientas especializadas de uso público para una auditoria, estableciendo las etapas de una auditoria y sus aspectos más significantes, el segundo realiza el análisis comparado para inferir la finalidad de los principales software con licencia pública general (GPL) utilizados en la auditoria, en la tercera sección determina el objeto de estudio, como algunos de los principales software GPL utilizados en auditoria, estos se analizan mediante una matriz de comparación, conforme a las principales características establecidas en un software de auditoria funcional. Del análisis de la información se concluye sobre la versatilidad del software revisados y se establecen conclusiones de su aplicabilidad.
\end{abstract}

Palabras clave - Auditoría, Técnicas, Herramientas, Planeación y Software libre. Términos.

Abstract - This article explores the results of a research project entitled "Impact of audit processes in the planning phase under concepts of specialized techniques of data analysis firms Ibagué " whose aim is to enable comparisons to make judgments about the use of free software for a specific application, which mythologically is developed through a comparative analysis between the tools or software with more public license used for the development of a financial audit.

Structurally consists of three sections, the first of which systematized literature techniques and specialized tools for public use for an audit, setting the stage for an audit and its most significant aspects, the second performs the comparative analysis to infer the purpose of the main software GPL (General Public License) used in audit, in the third section determines the object of study, these are analyzed by a matrix of comparison, according to the main characteristics in functional audit software
The analysis concludes information about the versatility of the revised software and conclusions on their applicability.

Key Word - Audit, Techniques, Tools, Planning and Free Software.

\section{INTRODUCCIÓN}

Los procesos de auditoría contable sirven para generar revisiones independientes a los estados financieros, estos son llevados a cabo por personas competentes con o sin la ayuda de software especializado para tal fin, a su vez emiten informes basados en los resultados producto de sus observaciones. Según [1]. Es un proceso sistemático para obtener y evaluar de manera objetiva las evidencias relacionadas con informes sobre actividades económicas y otros acontecimientos relacionados, cuyo fin consiste en determinar el grado de correspondencia del contenido informativo con las evidencias que le dieron origen. En general las empresas evidencian la necesidad de tener un control en el desarrollo de sus actividades para el cumplimiento de su objeto social, es aquí donde la auditoria pasa a ser una herramienta de evaluación que forma parte del proceso administrativo y operativo del sistema.

Con el propósito de dar valides a este proceso existen diversos métodos prácticos que son utilizados por la persona encargada de realizar las auditorias para la verificación y racionalidad de la información obtenida, estos son conocidos como técnicas de auditoría; entre las más utilizadas se tiene.

- El estudio general el cual aprovecha la experiencia del auditor para que basado en la observación de la empresa y constatando la información suministrada en los libros contables detecte comportamientos atípicos para un cargo determinado, particularmente cuando el nivel de importancia en la jerarquía del ente auditado es bajo, este tipo de operaciones pueden presumir algún tipo de sospecha y requieren especial cuidado al momento de iniciar una auditoria. 
- La técnica del análisis, esta técnica se basa en la comparación de los registros contables, con los índices, transacciones, variaciones y movimientos desarrollados en función de las expectativas propuestas para el periodo contable.

- La técnica de la comprobación, se basa en la verificación de la exactitud aritmética de la información de los registros contables, con el propósito de detectar inexactitudes.

- Preguntas de indagación, a partir de la interacción con las personas directamente involucradas con el departamento o ente auditado, es posible obtener información relevante sobre situaciones o hechos que ameriten un especial cuidado al momento de su revisión.

Estas técnicas aportan de manera importante al proceso de auditoría en todas sus etapas y pueden contar con el apoyo de herramientas informáticas para su ejecución y desarrollo, algunas de estas herramientas son licenciadas y otras de uso público. La licencia GPL (General Public License o licencia pública general) fue creada a mediados de los años 80 como un mecanismo para impulsar el uso de software libre. La licencia GPL obliga al licenciatario a hacer públicos los códigos fuente del software desarrollado, con el fin de permitir a los usuarios del mismo puedan hacer modificaciones, adaptaciones o mejoras al programa [2]. Los usuarios de este tipo de licencias tienen la obligación de licenciar bajo el esquema GPL los productos resultantes del cambio, mejora o utilización de software con licencia pública. La utilización de este tipo de licenciamiento ha fomentado el crecimiento del número de usuarios de estos programas y el desarrollo de estos paquetes GPL.

Como se hace evidente los actuales procedimientos contables en las empresas hacen necesario que los profesionales auditores se involucren con las novedades tecnológicas, afín de hacer frente a los procesos contables que se encuentran presentes en la mayoría de los sectores económicos este cumulo de información, requiere que la evaluación para la toma de decisiones sea más precisa y argumentada, para ello se cuenta con herramientas tecnológicas que requieren ser evaluadas para establecer su grado de confiablidad.

El objeto de este documento es presentar un numero diverso de paquetes contables licenciados como GPL o más comúnmente conocidos como software libre utilizados en los procesos de auditoría contable, generando comparaciones subjetivas en función del costo computacional, el ambiente de trabajo y los alcances de cada uno de estos paquetes, de esta forma permitir dar herramientas de juicio al momento de elegir una alternativa de aplicación en correspondencia de las necesidades del usuario.

\section{ETAPAS DE LA AUDITORIA}

Los procesos de auditoria comprenden una serie de etapas o fases que deben ser cumplidas en su totalidad con ellas se hace explicita la necesidad de obtener evidencia suficiente y competente que respalden la opinión del auditor, haciendo énfasis en lo contable [3]. Estas se pueden identificar con claridad.

La planeación. Es el paso inicial para realizar auditorías eficaces consiste en la comprensión del ambiente en que se desempeña la empresa y la organización, así como los procesos inherentes a la gestión de documentos; en esta etapa se identifican y determinan los criterios con los cuales se va a auditar. Para ello es necesario realizar una propuesta objetiva que involucre el reconocimiento de la entidad auditada, aquí se debe determinar la estructura de la organización, el sector empresarial al cual pertenece, la estructura de capital que posee, entre otros, esta información es posible obtenerla realizando búsquedas independientes, consultas personales o indagando en los libros contables, posterior a esto se debe estimar el objeto de la auditoria en el cual se determina el alcance de la misma y los usuarios potenciales de la información; con ello se debe ser especialmente precavido debido a que es posible generar conceptos errados debido a una inapropiada o escasa información, por ello es indispensable establecer la propuesta de ejecución procedimental que debe cubrir todos aquellos aspectos que involucran el desarrollo de la actividad, como son las horas investidas, el costo del personal externo, los equipos con los que se deba contar y los espacios dispuestos para la actividad. De forma general los principales elementos que componen esta etapa son:

- Reconocimiento de la entidad auditada

- Objeto de la auditoría

- Evaluación del riesgo

- Planificación del procedimiento

- Desarrollo de la actividad

La ejecución. Corresponde a la segunda etapa de una auditoría financiera aquí la información pasa por una serie de análisis para garantizar concordancia y razonabilidad en la misma, se realizan comparaciones entre los estados financieros, en búsqueda de evidencias o indicios sobre un particular, la revisión e interpretación, de los mismos, deben presentarse de manera clara con el propósito de establecer un soporte para la toma de decisiones por parte de los usuarios de la información, se debe prestar especial importancia en aquellas diferencias significantes con relación a los informes auditados, existen ocasiones en donde el tamaño de la entidad auditada es muy grande, para estos casos se hace necesario la utilización de métodos estocásticos que abarcan una muestra significativa que permite generar juicios justificados para la gerencia. Esta etapa se considera como el eje central del proceso de una auditoría y reviste especial importancia porque a partir de la información recolectada se procede establecer conclusiones sobre el trabajo ejecutado.

El informe. Corresponde a la etapa final del proceso de auditoría acá se debe establecer un concepto sobre los estados 
financieros de la entidad auditada, así como la estructura organizacional y de control con que cuenta, a partir de los indicios establecidos en la fase previa de ejecución se deben generar conclusiones objetivas y debidamente soportadas que pueden ser operativas o de fondo, estas últimas en particular para los casos en los que se ven involucradas personas y recursos deben justificarse minuciosamente y con argumentos en algunas ocasiones acompañados de recomendaciones en la operación

Las anteriores etapas forman parte del proceso de Auditoría normalmente denominada tradicional a partir de la información, reconocimiento y ejecución se espera, verificar la veracidad de la información financiera y establecer en cada caso la concordancia de la misma o la presunta irregularidad.

En la figura 1 se ilustran las etapas y los componentes más destacables de una auditoría financiera.
Conforme a [4-5-6]. La Auditoría contemporánea o de Gestión, propende por la evaluación multidisciplinaria, de riesgos y de procesos, adelantada en forma independiente y con un enfoque de sistemas, midiendo el grado y forma de cumplimiento de los objetivos de la organización y de la relación con su entorno; proponiendo alternativas para el logro más adecuado de sus fines y el mejor aprovechamiento de sus recursos.

Para ello es necesaria la inclusión de herramientas tecnológicas de soporte que ayuden a la organización y gestión de la información recopilada, así como proponer juicios sobre la misma que pueden llegar a ser una herramienta valiosa para los informes que se emitan.

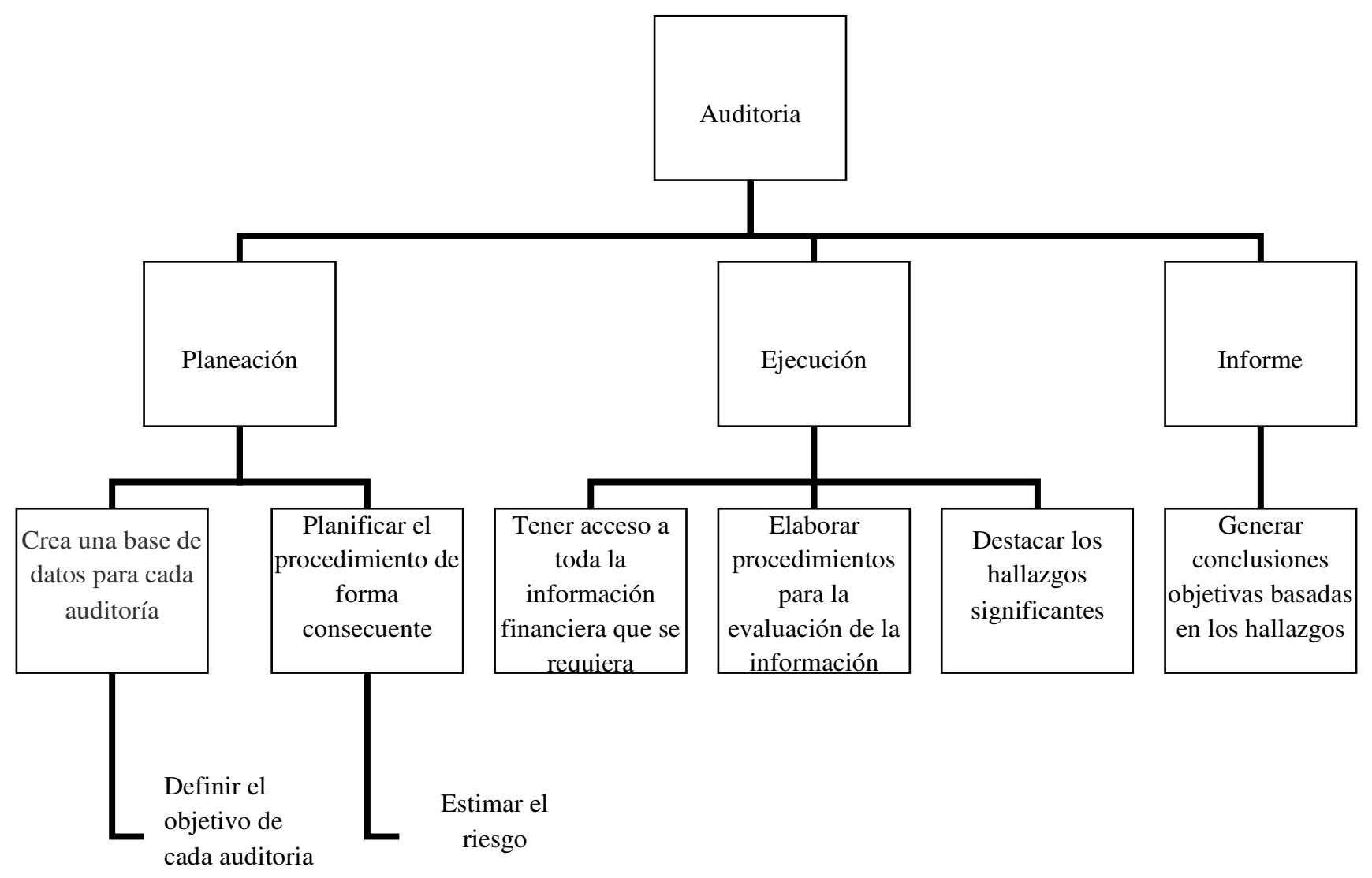

Figura 1. Etapas de una Auditoría Financiera (Fuente Propia) 


\section{HERRAMIENTAS CON LICENCIA PÚBLICA UTILIZADAS EN AUDITORÍA}

Las dinámicas financieras han planteado la necesidad de procesos de esparcimientos propios para estos escenarios y sus consecuentes, en esta perspectiva se han planteado diversos softwares con licencias GPL, con una variedad de características y campos de aplicación [7]. Todos ellos y conforme a la filosofía del libre uso deben exponer los códigos fuente, permitiendo la posibilidad de modificar y generar mejores adaptaciones a los mismos.

En la figura 2 se ilustran algunos del software más utilizados en auditorias financieras producto de una revisión adelantada dentro del marco del proyecto de investigación que acoge este artículo.

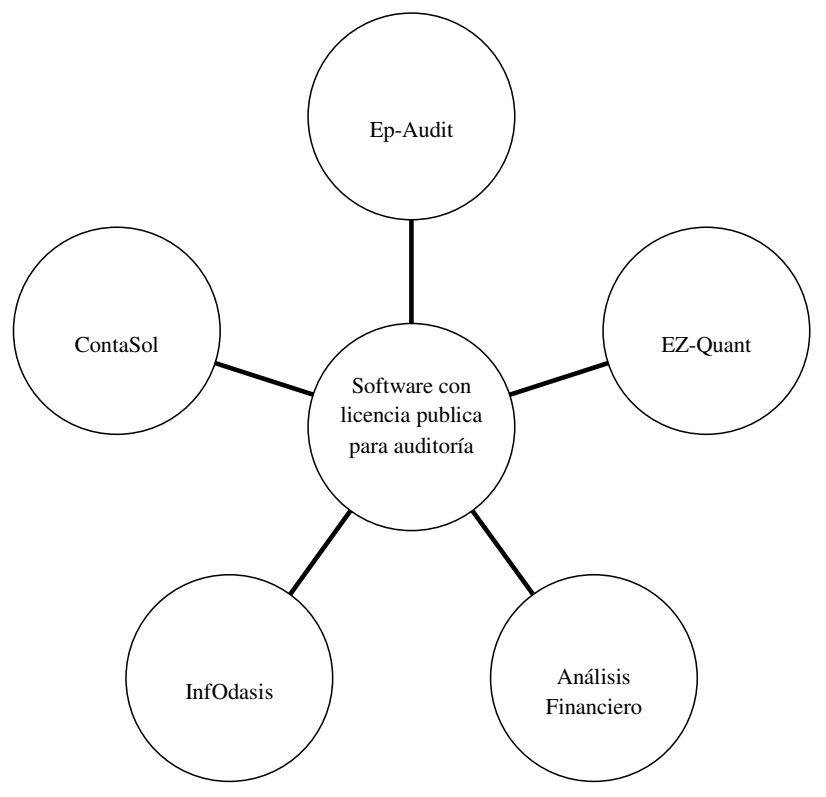

Figura 2. Software Libre Para Auditoría Financiera (Fuente Propia)

- Ep-Audit1, Este software se encuentra desarrollado con MS Office 2007 aprovechando toda su potencia. Se distribuye bajo licencia Creative Commons, su uso es gratuito y no tiene restricciones para hacer copias y distribuirlas, funciona bajo Access y se complementa con Excel y Word. Todos los conocimientos que posea de Access, Excel o Word le serán de utilidad para manejar esta aplicación aumentando la calidad y rapidez de su trabajo en auditoría.

\footnotetext{
${ }^{1}$ http://epaudit.blogspot.com/p/auditoria-financiera.html
}

- EZ-Quant2, Software "freeware" de muestreo estadístico desarrollado por la agencia "Defense Contract Audit Agency" (DCAA) aplicado a la auditoría, es un conjunto de tres aplicaciones estadísticas para realizar un muestreo estadístico, el análisis de regresión y análisis de gráficos.

- Análisis Financiero3, Programa de análisis económico y financiero desarrollado con MS Access, su principal función es el análisis y la evaluación de la información financiera de la empresa, su flexibilidad favorece la aplicación del mismo en empresas de tamaño diverso. Principalmente funciona bajo el principio de la comparación de la información financiera, de esta manera, consigue realizar una proyección de lo que sucederá en el ejercicio en curso. El programa divide su actividad en dos tareas principales, la captura de datos y el análisis de los mismos, los cuales se realizan de forma automática.

- InfOdasis4, Herramienta modular desarrollada bajo licencia GPL admite desarrollos a la medida, puede ser implementado en diferentes sectores empresariales, tiene la posibilidad de exportar la información de sus ficheros fuera del programa.

- ContaSol5, Herramienta desarrollada para el manejo de la contabilidad, especialmente dirigida a empresas de mediano tamaño. Posee como ventaja que no tiene límite en el número de usuarios al momento de utilizar el programa, así como con el número de empresas a introducir este programa, es compatible con Acess.

\subsection{Características del software con licencia publica revisados}

Con el propósito de establecer una comparación objetiva entre los softwares con licencia publica revisados, se han propuesto una serie de características deseables en un software de auditoria, las cuales se enuncian a continuación.

1. Tener la posibilidad de realizar un número ilimitado de auditorías con la aplicación.

2. Permitir generar una base de datos para cada proceso de auditoría.

3. Calcula los indicadores para el análisis económico y financiero.

4. Tener la capacidad de elaborar fichas, cuentas de movimientos, obtener balances y saldos de forma automática

\footnotetext{
2 http://www.dcaa.mil/ez_quant_applications.html

3 http://analisisfinanciero.todoaccess.com/Caracteristicas.asp

${ }^{4} \mathrm{http} / / / \mathrm{www}$.infodasis.com/web/index.php?id_section=101

5 http://gratis.portalprogramas.com/ContaSOL.html
} 
5. Realizar cruces de información financiera.

6. Capacidad de importar los libros de contabilidad.

7. Posibilidad de trabajar con diversas Unidades Monetarias.

8. Capacidad de contabilizar ajustes y reclasificaciones.

9. Destacar los hallazgos más relevantes.

10. Adecuada curva de aprendizaje y facilidad de mejoramiento de software.

En la Tabla 1 se realiza una comparación objetiva entre los softwares objeto de estudio, en función de las características previamente determinadas.

\begin{tabular}{|c|c|c|c|c|c|c|c|c|c|c|}
\hline & \multicolumn{10}{|c|}{ Característica } \\
\hline Software GPL & 1 & 2 & 3 & 4 & 5 & 6 & 7 & 8 & 9 & 10 \\
\hline Ep-Audit & 1 & b & 1 & 1 & b & b & l & b & b & l \\
\hline EZ-Quant & & 1 & & & & & l & 1 & 1 & 1 \\
\hline Análisis Financiero & & 1 & 1 & 1 & & l & l & l & l & \\
\hline InfOdasis & & l & l & l & l & b & b & l & l & l \\
\hline ContaSol & 1 & b & l & & 1 & 1 & 1 & 1 & 1 & \\
\hline
\end{tabular}

Tabla 1. Capacidad de cumplimiento de características del software revisado

En la Tabla 2 se muestra una comparación en función de la versatilidad o capacidad del software de cumplir un gran número de características deseables y la capacidad de interactuar con otros software o plataformas de uso reconocido.

\begin{tabular}{|c|c|c|}
\hline Software & Versatilidad & Interfaz Externa \\
\hline Ep- Audit & Alto & $\begin{array}{c}\text { Access, Excel o } \\
\text { Word }\end{array}$ \\
\hline EZ-Quant & Medio & Access \\
\hline Análisis & Alto & Access, Excel o \\
Financiero & Word \\
\hline InfOdasis & Alto & Access \\
\hline ContaSol & Bajo & \\
\hline
\end{tabular}

Tabla 2. Comparación de características del software revisado
Como es recomendable es muy conveniente contar con software compatibles con versiones comerciales, esto facilita la integralidad de la información.

\section{CONCLUSIONES}

Luego de haber realizado la caracterización de algunas de las herramientas con licencia publica más utilizadas en los procesos de auditoría, se plantean las siguientes conclusiones:

$>\quad$ Los procedimientos de auditoria realizados de forma consistente y bien formulada, deben establecer con certeza y objetividad la información con la mayor claridad y precisión, esto solamente es posible lograrlo cuando las etapas de la misma se han proyectado con rigor y profesionalismo.

$>\quad$ La planeación es una de las etapas más importantes del proceso de auditoría pues es en esta fase cuando se establecen los objetivos que pretende alcanzar y se determinan los mecanismos para lograrlo.

$>\quad$ Se ha determinado que los programas con licencia publica o software libre, son una herramienta de uso ampliamente difundido, además su filosofía hace que el gradiente de evolución sea positivo en la mayoría de los casos, lo cual garantiza un amplio desarrollo en los módulos que se operan.

$>\quad$ La utilización de herramientas tecnológicas, facilita el papel del auditor, es necesario establecer las características asociadas al software utilizados, con el propósito de seleccionar la mejor alternativa de uso, para un caso específico.

$>\quad$ Algunas herramientas como Ep-Audit e InfOdasis poseen una alta versatilidad lo cual permite ejecutar auditorias en múltiples escenarios financieros.

$>\quad$ La capacidad de lograr transferir información entre los softwares de licencia publica y programas de uso comercial es una característica deseable en la mayoría de los casos, esto a la vez potencia el interés de los usuarios en el desarrollo de la herramienta.

\section{AGRADECIMIENTOS}

Los autores agradecen a la Dirección de Investigaciones de la Universidad Cooperativa de Colombia Sede Ibagué, por el apoyo en la realización del proyecto de investigación, 'Impacto de procesos de auditoria en la fase de planeación bajo conceptos de técnicas especializadas de análisis de datos en las empresas de la ciudad de Ibagué'. 


\section{REFERENCIAS}

[1]. A. Martínez, A. Blanco, B. Yeiniel Briseida, L. Liuba, y L. Marichal, "Auditoría con Informática a Sistemas Contables," Revista de Arquitectura e Ingeniería, vol. 6, núm. 2, agosto, pp. 1-14, 2012

[2]. C. Galeano, J. Mantilla, C. Duque, y M. Mejía. "Herramientas de software con licencia pública general para el modelado por elementos finitos," Revista Dyna, vol. 74, núm. 153, noviembre, pp. 313-324, 2007.

[3]. C. Ocampo, O. Trejos, G. Solarte, "Las tecnicas forenses y la auditoría," Scientia et Technica, Año XVI, No 45, pp. 108-113, 2010.

[4]. O. Falconí, Oscar. "Auditoría y las Normas de Auditoría Generalmente Aceptadas, " Contabilidad y Negocios, Pontificia Universidad Católica del Perú, Año 1(2), pp. 16-20, 2006.

[5]. R. Triana. "La auditoría de gestión, una solución ante los retos provocados por los escándalos financieros," Escenarios, Vol. 8, No. 2, Julio-Diciembre, pp. 2130, 2010.

[6]. R. Zaldivar. "Auditoría como herramienta para el control de la gestión de los recursos humanos," Dimensión Empresarial, Vol. 7 No. 1, Enero - Junio, pp. 6-10, 2009.

[7]. K. Tarek, H. Abdel-Hamid, "The Economics of Software Quality Assurance: A Simulation Based Case Study," Management Information Systems Research Center, Vol. 12, No. 3 (September), pp. 395-411, 1988. 